\title{
Revealing the Nanoscale Structure and Chemistry of Intact Solid-Liquid Interfaces in Electrochemical Energy Storage Devices by Cryo-FIB Lift-Out and Cryo-STEM
}

Michael J. Zachman ${ }^{1}$, Zhengyuan $\mathrm{Tu}^{2}$, Lynden A. Archer ${ }^{2,3}$, and Lena F. Kourkoutis ${ }^{1,4}$

1. School of Applied and Engineering Physics, Cornell University, Ithaca, NY 14853, USA.

2. Department of Materials Science and Engineering, Cornell University, Ithaca, NY 14853, USA.

3. School of Chemical and Biomolecular Engineering, Cornell University, Ithaca, NY 14853, USA.

4. Kavli Institute for Nanoscale Science, Cornell University, Ithaca, NY 14853, USA.

Solid-liquid interfaces play a critical role in a range of energy capture and storage devices, but often lack high-resolution characterization with the liquids intact. For example, lithium metal batteries (LMBs) offer ten times the anode storage capacity of lithium-ion batteries, but are limited by capacity fade and safety hazards due to processes at the anode-electrolyte interface [1]. These processes, breakdown of electrolyte to form a "solid-electrolyte interphase" (SEI) layer and uneven deposition of lithium metal leading to dendrite growth, are highly interrelated [2,3]. Understanding the formation and composition of SEI layers is therefore critical to controlling these processes, and will require new techniques to resolve the structural and chemical features of nanoscale SEIs at intact interfaces taken from devices.

Scanning transmission electron microscopy (STEM) allows atomic scale characterization of structure and bonding [4], but is traditionally limited to hard materials like solid-solid interfaces due to the volatility of liquids in the microscope vacuum. In biology, cryo-transmission electron microscopy (cryoTEM) is an established technique for characterizing the native structure of specimens such as molecules or small cells snap frozen in thin aqueous films [5], but it has seen limited applications in other fields due to sample size constraints. Recently, we have demonstrated cryo-focused ion beam (cryo-FIB) liftout for preparation of electron transparent cross-sections from bulk samples or devices with internal solid-liquid interfaces stabilized by rapid freezing [6].

Here, we demonstrate the unique ability of cryo-FIB lift-out combined with analytical cryo-STEM to provide nanoscale structural and chemical information about intact solid-liquid interfaces from energy storage devices. To do this, we prepare cross-sections of anode-electrolyte and dendrite-electrolyte interfaces from cycled LMB coin cells by cryo-FIB lift-out (Fig. 1). Using cryo-STEM dark-field imaging and electron energy loss spectroscopy (EELS) in combination with multivariate curve resolution we then directly observe structure and track local bonding states across these interfaces, revealing two distinct chemical bonding environments largely segregated in the SEI and electrolyte (Fig. 2). This provides the first nanoscale compositional information about intact SEI layers in LMBs, showing how cryo-FIB lift-out and analytical cryo-STEM can lead to new insights into processes such as SEI and dendrite formation at solid-liquid interfaces in electrochemical energy storage devices [7].

[1] J.-M. Tarascon and M. Armand, Nature 414 (2001), p. 359.

[2] P. Bai et al, Energy Environ. Sci. 9 (2016), p. 3221.

[3] A. Kushima et al, Nano Energy 32 (2017), p. 271.

[4] D.A. Muller et al, Science 319 (2008), p. 1073.

[5] J. Dubochet et al, Q. Rev. Biophys. 212 (1988), p. 129.

[6] M. Zachman et al, Microsc. Microanal. 22 (2016), p. 1338.

[7] This work was supported by NSF (DMR-1654596) and the Packard Foundation. It made use of the CCMR Shared Facilities which are supported through the NSF MRSEC program (DMR-1120296). 

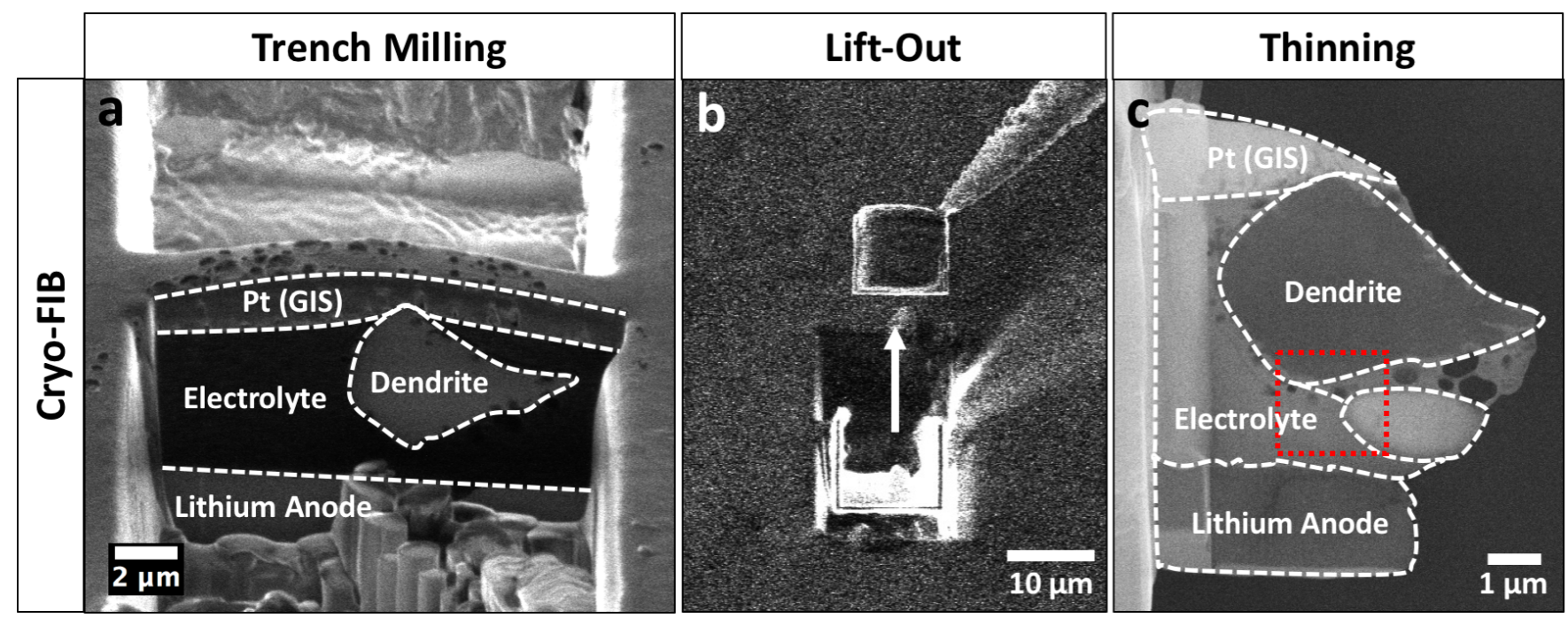

Figure 1. Preparation of intact solid-liquid interfaces from a lithium metal battery (LMB) for cryoscanning transmission electron microscopy (cryo-STEM) by cryo-focused ion beam (cryo-FIB) lift-out. Coin cell batteries are manually opened and immediately snap frozen, preserving the electrolyte. (a) The frozen coin cell is transferred into the cryo-FIB and the ion beam is used to site-specifically mill trenches on either side of a structure of interest, leaving a thin vertical lamella. Here, the lamella contains a cross-section of a dendrite growing horizontally above the anode. (b) Water vapor deposition used to attach the lamella to a cooled nanomanipulator, the lamella is freed from the device with the ion beam, and lifted out. (c) The lamella is attached to a TEM lift-out grid with water vapor deposition, the needle is cut free, and the ion beam is used to thin the sample to electron transparency.

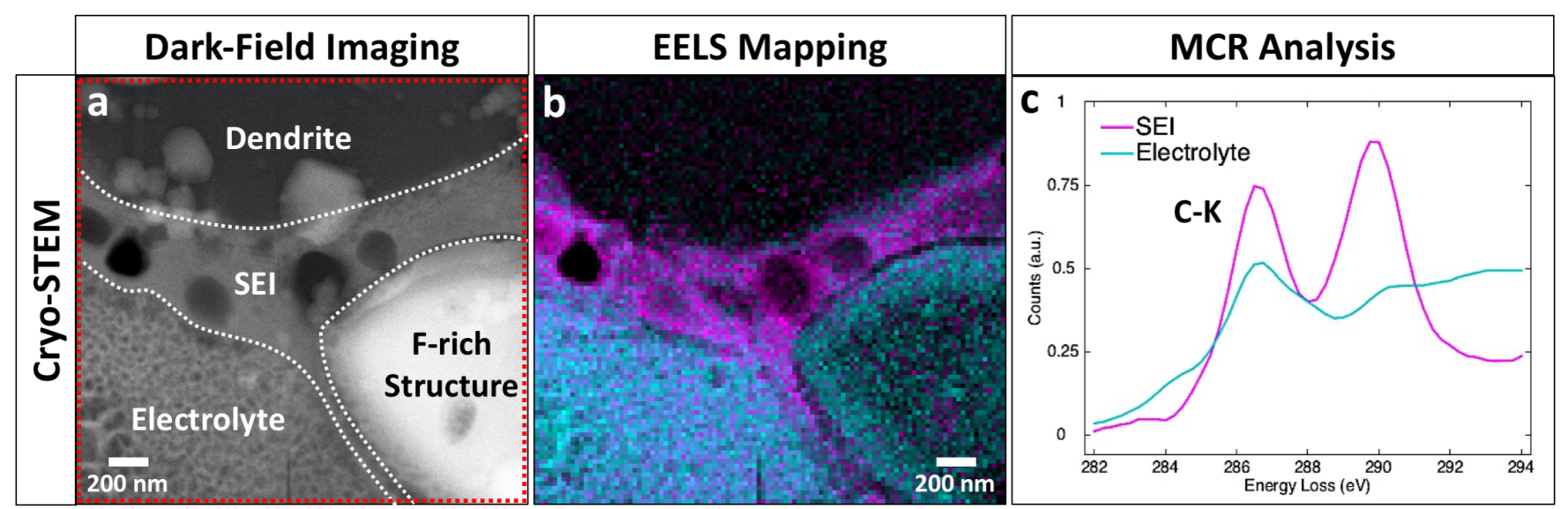

Figure 2. Mapping of the local structure and carbon bonding state across an intact solid-liquid interface at the surface of a dendrite in a LMB prepared by cryo-focused ion beam (cryo-FIB) lift-out. (a) Nanoscale annular dark-field cryo-STEM imaging of the region marked in red in Figure 1 revealed a thick SEI layer at the dendrite-electrolyte interface. (b) Carbon EELS mapping and multivariate curve resolution (MCR) analysis of this region clearly resolved two spectral components largely segregated in the electrolyte and the SEI, with distinct local bonding environments (c). 